\title{
A shift in macrophage phenotype underlies age-related ENS degeneration
}

An age-dependent shift in macrophage polarization to a pro-inflammatory phenotype has been shown in a new study to cause inflammation-dependent degeneration of the enteric nervous system (ENS) in mice.

Degenerative changes to the ENS and loss of enteric neurons occurs with age, but the process is poorly understood. To find out more about age-associated ENS degeneration, the researchers examined the longitudinal muscle and myenteric plexus of the small intestine from young ( 3 months), middle-aged (10-12 months) and old (20-24 months) mice, as well as characterized the muscularis macrophages (MMs).

Becker et al. found that ageing caused a shift in the MMs in the mice from an anti-inflammatory $\mathrm{M} 2$ phenotype to a pro-inflammatory M1 phenotype. This shift was associated with chronic low-grade inflammation in the ENS microenvironment, increased apoptosis and loss of enteric neurons and enteric neural stem cells, and delayed intestinal transit.

"The change in $[\mathrm{MM}]$ phenotype is partly a phenomenon intrinsic to macrophages and was related to an age-dependent decline in expression of the transcription factor FOXO3," explains author Laren Becker. Interestingly, FOXO3-deficient mice had signs of the premature ageing phenotype of the ENS. The investigators hope to confirm their findings in humans as targeting MMs could be a potential approach to reduce inflammation and combat age-associated degeneration of the gut.

Katrina Ray

ORIGINAL ARTICLE Becker, L. et al. Age-dependent shift in macrophage polarisation causes inflammation-mediated degeneration of enteric nervous system. Gut http://dx.doi.org/10.1136/gutjnl-2016-312940 (2017) 